\title{
Effects of exenatide on circulating glucose, insulin, glucagon, cortisol and catecholamines in healthy volunteers during exercise
}

\author{
E. Y. H. Khoo • J. Wallis $\cdot$ K. Tsintzas • \\ I. A. Macdonald • P. Mansell
}

Received: 19 August 2009/Accepted: 24 September 2009/Published online: 7 November 2009

(C) Springer-Verlag 2009

\begin{abstract}
Aims/hypothesis Exenatide, a glucagon like peptide-1 agonist, is a treatment for type 2 diabetes mellitus that stimulates insulin and suppresses glucagon secretion in a glucose-dependent manner. By contrast, during aerobic exercise, the serum insulin concentration normally falls, with a rise in plasma glucagon. We therefore assessed whether exenatide might predispose to hypoglycaemia during exercise.

Methods We studied eight non-diabetic men, who were $35.3 \pm 6.3$ years of age with BMI of $24.7 \pm 1.7 \mathrm{~kg} / \mathrm{m}^{2}$ (mean $\pm \mathrm{SD}$ ), using a randomised, crossover, double-blind design investigation. After an overnight fast, participants received $5 \mu \mathrm{g}$ of subcutaneous exenatide or placebo and rested for $105 \mathrm{~min}$ before cycling at $60 \%$ of their maximal oxygen uptake $\left(\dot{V} \mathrm{O}_{2 \max }\right)$ for $75 \mathrm{~min}$ and then recovering for a further $60 \mathrm{~min}$.
\end{abstract}

E. Y. H. Khoo $\cdot$ P. Mansell $(\bowtie)$

Department of Diabetes and Endocrinology,

Nottingham University Hospitals,

Queens Medical Centre Campus, Derby Road,

Nottingham NG7 2UH, UK

e-mail: Peter.Mansell@nuh.nhs.uk

E. Y. H. Khoo $\cdot$ J. Wallis $\cdot$ K. Tsintzas $\cdot$ I. A. Macdonald

P. Mansell

School of Biomedical Sciences, University of Nottingham,

Nottingham, UK

Present address:

E. Y. H. Khoo

Division of Endocrinology, Department of Medicine,

National University of Singapore,

Singapore, Republic of Singapore
Results The insulin/glucagon molar ratio rose with exenatide at rest $(p<0.01)$, then fell during exercise with placebo and with exenatide. At rest, fasting blood glucose fell by approximately $1 \mathrm{mmol} / 1$ with exenatide to a nadir of $3.4 \pm$ $0.1 \mathrm{mmol} / \mathrm{l}(p<0.01)$. During exercise, blood glucose fell with placebo but, unexpectedly, rose with exenatide. Plasma adrenaline (epinephrine) and noradrenaline (norepinephrine), but not cortisol concentrations increased to a greater extent during exercise after exenatide. No participant developed symptomatic hypoglycaemia and the lowest individual blood glucose recorded was $2.8 \mathrm{mmol} / \mathrm{l}$ with exenatide at $50 \mathrm{~min}$ in the pre-exercise period.

Conclusions/interpretation In non-diabetic participants given exenatide, blood glucose concentrations rise rather than fall during aerobic exercise with an associated greater catecholamine response.

Keywords Exenatide · Exercise - GLP-1 - Glucagon-like peptide- $1 \cdot$ Hypoglycaemia

$\begin{array}{ll}\text { Abbreviations } \\ \text { IGMR } & \text { Insulin glucagon molar ratio } \\ \dot{V} \mathrm{O}_{2} & \text { Oxygen uptake } \\ \dot{V} \mathrm{O}_{2 \max } & \text { Maximal oxygen uptake }\end{array}$

\section{Introduction}

Exenatide, a glucagon-like peptide-1 mimetic, has several beneficial modes of action for patients with type 2 diabetes [1]. Exenatide increases satiety, delays gastric emptying, increases insulin and decreases glucagon concentrations. The endocrine responses are glucose-dependent [1], although there is still a pronounced insulinotropic effect at glucose concentrations of 
5.0 and $5.4 \mathrm{mmol} / \mathrm{l}[2]$ with a modest fall in fasting blood glucose concentration in non-diabetic volunteers [3]. The glucose-dependence of the endocrine effects explains the low risk of hypoglycaemia with exenatide in clinical studies [4].

In direct contrast to exenatide, aerobic exercise leads to a fall in serum insulin and a rise in glucagon concentrations, which protect against a severe decline in blood glucose levels. Exenatide might therefore predispose to hypoglycaemia during exercise by preventing the decrease in the insulin/ glucagon ratio that is normally observed. Alternatively, if there is no such effect, this suggests a potential advantage of therapy with exenatide over sulfonylureas and insulin, which are both associated with hypoglycaemia during exercise.

We studied the effects of a single dose of exenatide on blood glucose concentrations and hormone responses to exercise in non-diabetic participants.

\section{Methods}

This was a single-centre, randomised, placebo-controlled, double-blinded, crossover study, approved by the Ethics Committee of the University of Nottingham and conducted in accordance with the Helsinki Declaration, with participants giving written informed consent.

Participants We recruited eight healthy, male volunteers aged $35.3 \pm 6.3$ years with BMI $24.7 \pm 1.7 \mathrm{~kg} / \mathrm{m}^{2}$ and percentage body fat $17.4 \pm 3.7 \%$ (mean $\pm \mathrm{SD}$ ) as estimated by bio-impedance (Quadscan 4000; BodyStat, Douglas, Isle of Man, UK).

Preliminary and study session visits Participants attended two preliminary visits. First, they were observed for $3 \mathrm{~h}$ following a test injection of $5 \mu \mathrm{g}$ exenatide after a $10 \mathrm{~h}$ overnight fast. No participants developed symptomatic hypoglycaemia or significant nausea at rest. At the second preliminary visit, participants familiarised themselves with the electronically-braked cycle ergometer (Lode Excalibur; Lode, Groningen, the Netherlands) and the respiratory gas exchange apparatus (Vmax 29; Sensormedics, Yorba Linda, CA, USA). During the study session, participants underwent an incremental work test to determine maximal oxygen uptake $\left(\dot{V} \mathrm{O}_{2 \max }\right)$ as defined by the British Association of Sport and Exercise Sciences Criteria [5]. Mean $\dot{V} \mathrm{O}_{2 \max }$ was $40.0 \pm 4.9 \mathrm{ml} \mathrm{kg}^{-1} \mathrm{~min}^{-1}$. The experimental work rate was established by calculating $60 \%$ of each participant's $\dot{V} \mathrm{O}_{2 \max }$ from the oxygen uptake $\left(\dot{V} \mathrm{O}_{2}\right)$-workload relationship.

Randomisation Participants were randomised using the website www.randomization.com (accessed 1 June 2008).

Experimental design In each study session, following a $10 \mathrm{~h}$ fast, a retrograde cannula was inserted into the dorsal hand vein and the participant's hand was placed in a warm air box for arterialised blood sampling. Throughout each study session, blood was sampled regularly for immediate determination of glucose and lactate and later analysis for insulin, glucagon, cortisol, NEFA and catecholamine concentrations. Participants received a subcutaneous injection of either $5 \mu \mathrm{g}$ exenatide or placebo (at time 0 ) and were initially observed resting supine. Exercise commenced after $105 \mathrm{~min}$ to coincide with the projected peak serum exenatide concentration [6]. Participants then cycled for $75 \mathrm{~min}$ and regular measurements of $\dot{V} \mathrm{O}_{2}$ confirmed the mean exercise intensity to be $65.5 \%$ and $64.9 \%$ of $\dot{V} \mathrm{O}_{2 \max }$ with exenatide and placebo respectively. Participants' perceptions of the intensity of exercise were recorded using the Borg scale [7]. After exercise, participants transferred back to the couch to recover from 180 to $240 \mathrm{~min}$. Participants returned after 1 to 3 weeks for the crossover arm of the study.

Assays Whole-blood glucose and lactate were measured with an analyser (2300 Stat Plus-D; Yellow Springs Instruments, Yellow Springs, OH, USA). Serum insulin and plasma glucagon were measured using double antibody radioimmunoassays (Diagnostic Products, Llanberis, UK). Catecholamines were determined by high-performance liquid chromatography with electrochemical detection [8]. Plasma NEFA and serum cortisol were measured using commercially available test kits (NEFA C; Wako, Germany and R\&D Systems, UK respectively). The cortisol assay was modified in house using spiking of parallel samples to allow for apparent incomplete recovery. Plasma exenatide concentrations were measured by an ELISA method by Amylin Pharmaceuticals, San Diego, CA, USA.

The insulin/glucagon molar ratio (IGMR) was calculated according to the modified formula of Muller et al. [9]: (insulin $[\mathrm{pmol} / 1] /$ glucagon $[\mathrm{pg} / \mathrm{ml}]$ ) $\times 3.26=$ IGMR .

Statistical analysis Data are expressed as mean \pm SE unless otherwise specified. Statistical analysis was performed using SPSS, version 15 (SPSS, Chicago, IL, USA). Data obtained with exenatide and placebo were compared using two-way (treatment, time) ANOVA with repeated measures. When significant treatment $\times$ time interactions were detected, selected point data were compared using paired $t$ tests with $p$ values adjusted using the Bonferroni correction. A value of $p<0.05$ indicated significant differences. NS signifies no significant differences between groups.

\section{Results}

All eight participants completed the control study. With exenatide treatment, one participant became nauseous, 
vomited and stopped after $50 \mathrm{~min}$ of exercise, his data thereafter were excluded. His lowest plasma glucose concentration was $3.9 \mathrm{mmol} / \mathrm{l}$.

Symptoms The Borg score increased to a median of 15 (hard) at the end of exercise with both exenatide and placebo (NS). No participant described symptoms attributable to hypoglycaemia.

Blood glucose profile As shown in Fig. 1, with exenatide the fasting blood glucose concentration fell from $4.5 \pm 0.1$ to $3.4 \pm 0.1 \mathrm{mmol} / \mathrm{l}$ at $55 \mathrm{~min}$, before rising to $3.8 \pm 0.1 \mathrm{mmol} / 1 \mathrm{just}$ prior to exercise; by comparison, little variation was seen with placebo ( $p=0.001$ for treatment $\times$ time interaction). During exercise, there was a treatment $\times$ time interaction $(p=0.007)$, with blood glucose falling by $0.5 \pm 0.1 \mathrm{mmol} / \mathrm{l}$ as expected with placebo, but rising by $0.4 \pm 0.1 \mathrm{mmol} / 1$ with exenatide $(p<0.0005)$. During recovery, blood glucose levels converged towards the end of the study $(p=0.012$ for treatment $\times$ time interaction). The lowest individual blood glucose observed in the entire study was $2.8 \mathrm{mmol} / \mathrm{l}$ with exenatide at $50 \mathrm{~min}$ (during resting phase).

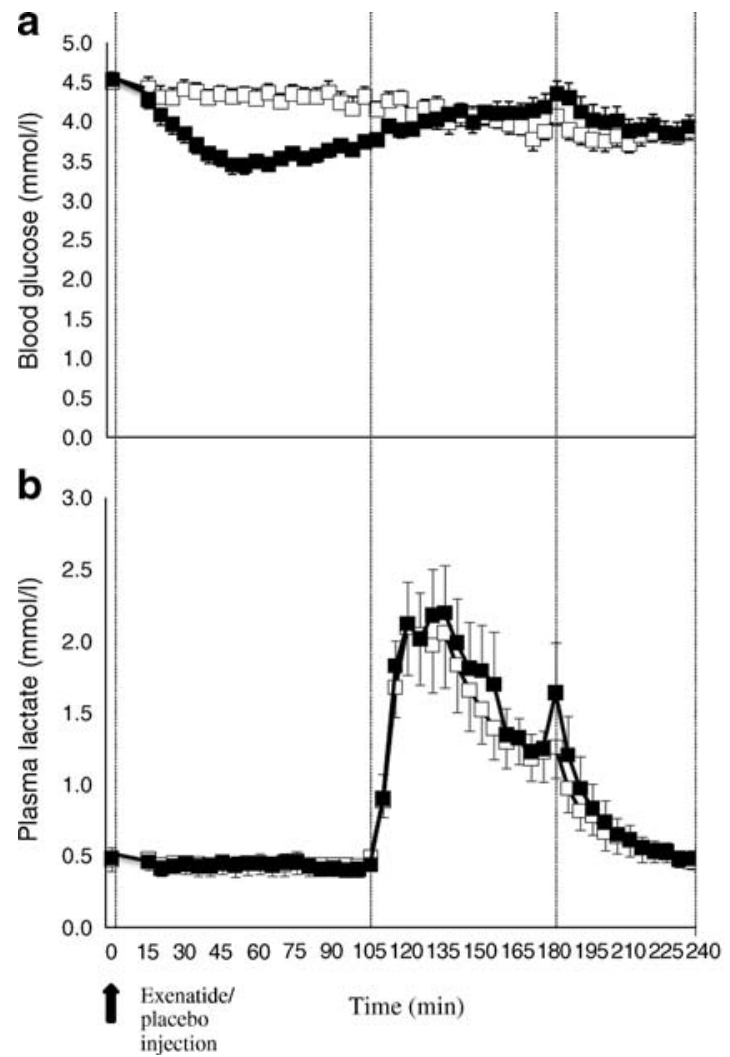

Fig. 1 Blood glucose (a), lactate (b), NEFA (c) and plasma exenatide (d) profiles. Subcutaneous injection of exenatide or placebo was at $0 \mathrm{~min}$, resting phase from 0 to $105 \mathrm{~min}$, exercise phase at $60 \% \dot{V} \mathrm{O}_{2 \max }$ from 105 to $180 \mathrm{~min}$ and the recovery phase was from 180 to $240 \mathrm{~min}$
Blood lactate As expected, blood lactate concentrations increased during exercise with peak values of approximately $2 \mathrm{mmol} / \mathrm{l}$ after 20 to $40 \mathrm{~min}$ for exenatide and placebo (NS; Fig. 1).

Plasma NEFA An expected rise in plasma NEFA concentrations was observed during exercise, with no significant differences between treatments (Fig. 1).

Plasma exenatide As predicted (and seen in Fig. 1), plasma exenatide levels were maximal between 60 and $120 \mathrm{~min}$ after injection.

Insulin and glucagon concentrations and insulin/glucagon molar ratio Figure $2 \mathrm{a}-\mathrm{c}$ shows that there was a trend towards higher insulin and lower glucagon concentrations with exenatide over the whole study, but no significant differences in either. As expected, the serum insulin concentration fell and glucagon increased with exercise.

With exenatide, IGMR rose from $1.66 \pm 0.19$ to $2.47 \pm 0.32$ at $30 \mathrm{~min}$, before falling to $1.58 \pm 0.40$ just prior to exercise; in contrast, little change was seen with placebo $(p=0.006$ for

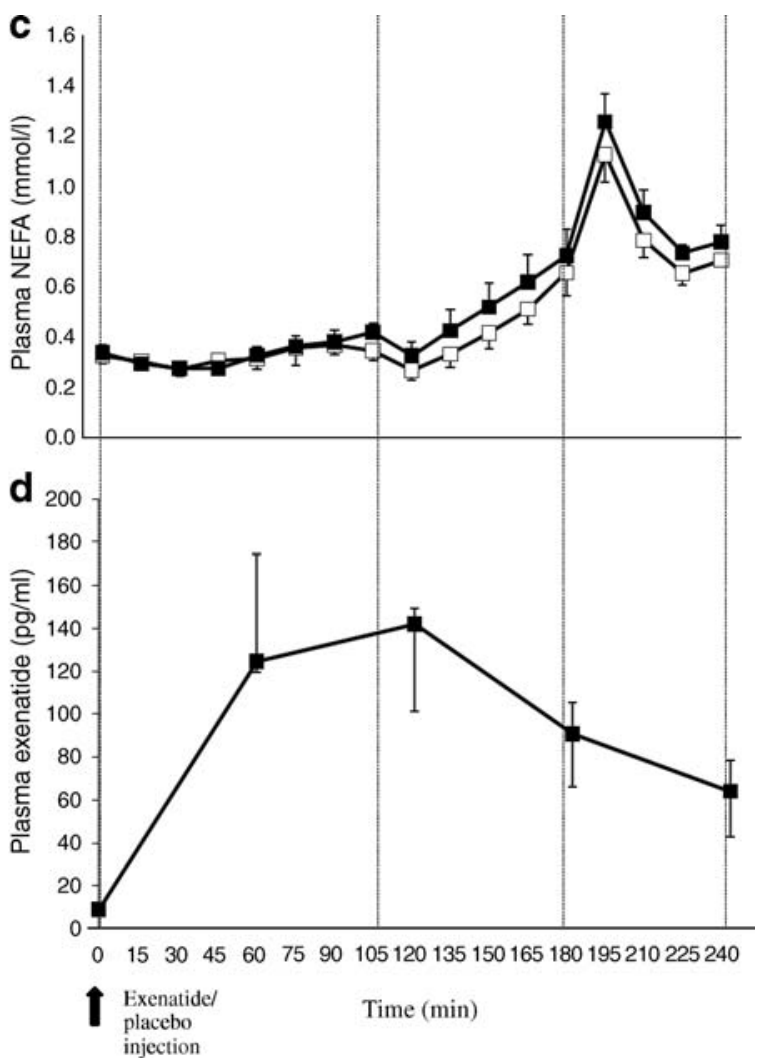

(phases indicated by dotted vertical lines). White squares, placebo; black squares, exenatide treatment. Data are mean $\pm \mathrm{SE}$ or $(\mathbf{d})$ median (interquartile ranges) 


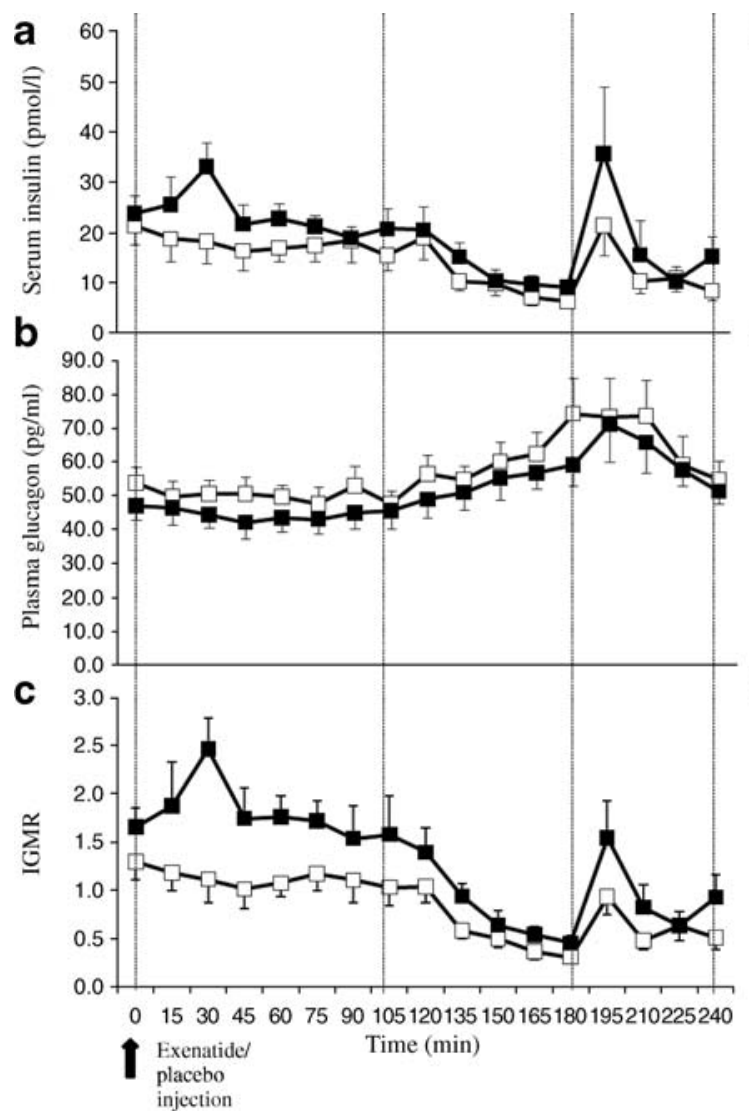

Fig. 2 Serum insulin (a), plasma glucagon (b), IGMR (c), serum cortisol (d), plasma noradrenaline (e) and plasma adrenaline (f) profiles. Subcutaneous injection of exenatide or placebo was at $0 \mathrm{~min}$, resting phase from 0 to $105 \mathrm{~min}$, exercise phase at $60 \%$

treatment effect). During exercise, IGMR fell with exenatide and control from $1.58 \pm 0.40$ to $0.46 \pm 0.08(p=0.031)$ and $1.03 \pm 0.19$ to $0.31 \pm 0.05(p=0.005)$ respectively, with no significant difference between groups. During the recovery phase, IGMR tended to be higher with exenatide $(p=0.076$ for treatment effect).

Serum cortisol A significant treatment $\times$ time interaction was seen in serum cortisol over the whole study ( $p=0.043$ ANOVA; Fig. 2d). It was principally due to a rise with exenatide and a fall with placebo during the resting phase ( $p=0.010$ for treatment $\times$ time interaction). There was no difference in the incremental increase in cortisol concentrations between exenatide and placebo treatments during exercise.

Plasma catecholamine concentrations As seen in Fig. 2e, f, there were no differences in catecholamine concentrations during the resting phase. Adrenaline (epinephrine) and noradrenaline (norepinephrine) concentrations increased during exercise with different responses after exenatide ( $p=0.019$ treatment $\times$ time interaction for adrenaline; $p=0.03$ for treatment $\times$ time interaction noradrenaline). At $135 \mathrm{~min}$,

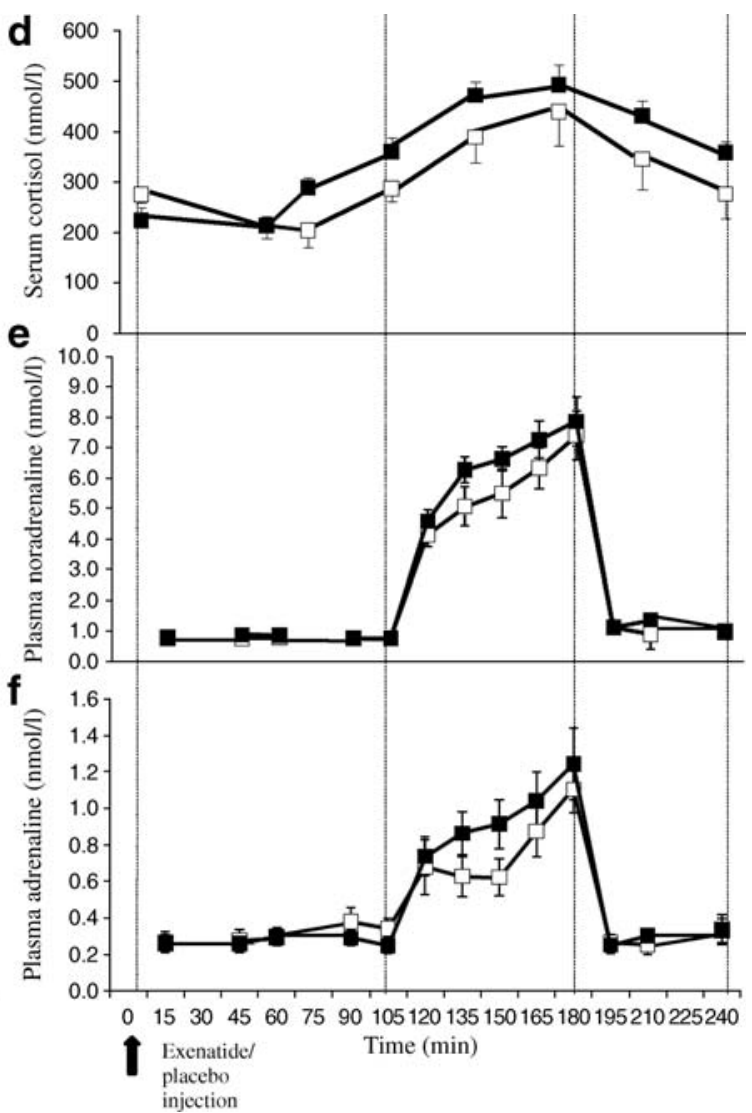

$\dot{V} \mathrm{O}_{2 \max }$ from 105 to $180 \mathrm{~min}$ and the recovery phase was from 180 to $240 \mathrm{~min}$ (phases indicated by dotted vertical lines). White squares, placebo; black squares, exenatide treatment. Data are means $\pm \mathrm{SE}$

there was a significantly greater increase in adrenaline concentration with exenatide $(p=0.01)$.

\section{Discussion}

Exenatide did not cause symptomatic hypoglycaemia in nondiabetic volunteers undergoing moderately intense aerobic exercise. Although the fasting blood glucose concentration fell by approximately $1 \mathrm{mmol} / \mathrm{l}$ after exenatide, it subsequently rose during exercise to concentrations above those seen with placebo. The Borg scales suggest that exenatide did not affect the subjective difficulty of undertaking exercise.

We chose to use a single subcutaneous dose of $5 \mu \mathrm{g}$ exenatide. The maintenance dose for treating patients with type 2 diabetes mellitus is $10 \mu \mathrm{g}$ twice daily, but in a pilot study we found that $10 \mu \mathrm{g}$ caused unacceptable nausea and even vomiting during exercise. Although intravenous exenatide $[2,10]$ would enable a steady-state concentration of exenatide, we wished to approach the 'real-life' situation as far as possible with a subcutaneous injection. The plasma exenatide concentrations achieved here are consistent with other published data 
[6] and the maximum concentration was observed at $120 \mathrm{~min}$, confirming that exercise took place at peak exenatide levels and that the effects persisted throughout the study.

With placebo, blood glucose fell at the onset of exercise as predicted. The increase in blood glucose during exercise with exenatide was unexpected. The IGMR fell during exercise in both groups, but tended to be higher after exenatide, and so differences in insulin and glucagon concentrations cannot alone explain the different patterns of change in blood glucose concentration. An alternative explanation for the rise in blood glucose during exercise with exenatide relates to the increased catecholamine concentrations, suggesting enhancement of sympatho-adrenal activity and potentially increased hepatic glucose output. In contrast, other investigators $[2,10]$ have not shown any difference in catecholamine responses to hypoglycaemia with exenatide.

Exenatide therefore does not induce symptomatic hypoglycaemia in healthy volunteers undergoing aerobic exercise. The present results cannot necessarily be extrapolated to the diabetic population.

Acknowledgements Nottingham University Hospitals Special Trustees generously provided funding for the hormone assays. Amylin Pharmaceuticals kindly undertook the analyses for plasma exenatide concentrations, but were not otherwise involved in sponsoring or designing study. S. Cordon of the School of Biomedical Sciences, University of Nottingham, Nottingham, UK analysed the hormone concentrations.

Duality of interest The authors declare that there is no duality of interest associated with this manuscript.

\section{References}

1. Nielsen LL, Baron AD (2003) Pharmacology of exenatide (synthetic exendin-4) for the treatment of type 2 diabetes. Curr Opin Investig Drugs 4:401-405

2. Degn KB, Brock B, Juhl CB et al (2004) Effect of intravenous infusion of exenatide (synthetic exendin-4) on glucose-dependent insulin secretion and counterregulation during hypoglycemia. Diabetes 53:2397-2403

3. Edwards CM, Stanley SA, Davis R et al (2001) Exendin-4 reduces fasting and postprandial glucose and decreases energy intake in healthy volunteers. Am J Physiol-Endocrinol Metab 281:E155E161

4. Kendall DM, Riddle MC, Rosenstock J et al (2005) Effects of exenatide (exendin-4) on glycemic control over 30 weeks in patients with type 2 diabetes treated with metformin and a sulfonylurea. Diabetes Care 28:1083-1091

5. Bird S, Davison R (1997) Guidelines for the physiological testing of athletes, 3rd edn. British Association of Sport and Exercise Sciences, Leeds, pp 59-72

6. Calara F, Taylor K, Han J et al (2005) A randomized, open-label, crossover study examining the effect of injection site on bioavailability of exenatide (synthetic exendin-4). Clin Ther 27:210-215

7. Borg GA (1982) Psychophysical bases of perceived exertion. Med Sci Sports Exerc 14:377-381

8. Forster CD, Macdonald IA (1999) The assay of the catecholamine content of small volumes of human plasma. Biomed Chromatogr 13:209-215

9. Muller WA, Faloona GR, Unger RH (1971) The influence of the antecedent diet upon glucagon and insulin secretion. N Engl J Med 285:1450-1454

10. Nauck MA, Heimesaat MM, Behle K et al (2002) Effects of glucagon-like peptide 1 on counterregulatory hormone responses, cognitive functions, and insulin secretion during hyperinsulinemic, stepped hypoglycemic clamp experiments in healthy volunteers. J Clin Endocrinol Metab 87:1239-1246 